\title{
Observer design for phenotypic observation of genetic processes
}

\author{
I. López $^{\mathrm{a}}$ M. Gámez ${ }^{\mathrm{a}}$ Z. Varga ${ }^{\mathrm{b}}$ \\ ${ }^{a}$ Department of Statistics and Applied Mathematics, University of Almería, Spain \\ ${ }^{\mathrm{b}}$ Institute of Mathematics and Informatics, Szent István University, Gödöllô, \\ Hungary
}

\begin{abstract}
In the paper a single-locus sexual population is considered, where the phenotypic selection process is described by an evolutionary game. First, in order to recover the genetic process from different observations, observability is guaranteed by the linearization method developed in earlier papers of the authors for systems with invariant manifold. Then, based on a known result of nonlinear systems theory, an observer system is constructed that makes it possible to asymptotically recover the solution of the original system from the observation. In the numerical illustrations the selection is described by a "rock-scissors-paper" type game widely studied in evolutionary game theory. For the corresponding evolutionary dynamics a Hopf bifurcation result is also obtained.
\end{abstract}

Key words: sexual population, evolutionary game, Hopf bifurcation, observer system

\section{Introduction}

In the applications of mathematical systems theory, the reconstruction of the state process from available measurements is an important issue for several

Email addresses: milopez@ual.es corresponding author, tel: (34)950015775, fax:(34)950015167 (I. López), mgamez@ual.es (M. Gámez), Varga.Zoltan@gek.szie.hu (Z. Varga). 
reasons. In biology, this problem naturally arises in relation with phenotypic observation of genetic processes. In static situation, in Garay and Garay (1998) biological conditions were given for the allele frequency - phenotype frequency correspondence to be one-to-one. In a dynamical situation, less restrictive conditions can guarantee that from the observation of time-dependent phenotypic characteristics the allele process can be uniquely recovered. In engineering practice, under the condition of observability and stability, for the effective approximate calculation of the state process from the observation, an auxiliary system, the so-called observer is constructed, the solution of which asymptotically produces the state process of the original system, in most cases with an exponential rate of convergence.

In earlier papers (López et al., 2006a, 2006b) observers have been constructed for different density-dependent population system models. In these models, for an observability analysis, it was enough to apply the classical sufficient condition of Lee and Markus (1971) for local observability. Since for frequencydependent selection processes the dynamic model has an invariant manifold, in order to guarantee local observability, we need to apply the linearization method developed in Varga (1992) for systems with invariant manifold. To effectively recover the genetic process from the phenotypic observation, an observer system will be designed, applying the results of Sundarapandian (2002). In the next section we shortly recall the basic concepts and theorems of the above observability and observer design methodology. In Section 3 evolutionary dynamics for an evolving sexual population is set up. In Section 4 a Hopf bifurcation for the evolutionary dynamics is obtained. Section 5 is dedicated to the observer design for two different phenotypic observation situations in the considered evolutionary model.

\section{Local observability and observer design}

Definition 2.1. For given $n \in \mathbb{N}$ with $n>1$, and $k \in \overline{1, n-1}$ a nonempty set $M \subset \mathbb{R}^{n}$ is called a regular $k$-dimensional submanifold, if there exist an open set $G \subset \mathbb{R}^{n}$ and a function $\phi \in C^{1}\left(G, \mathbb{R}^{n-k}\right)$ such that, for all $x \in G$, for the range of the derivative $\phi^{\prime}(x)$, we have $R_{\phi^{\prime}(x)}=\mathbb{R}^{n-k}$ and $M=\phi^{-1}(0)$.

For a continuously differentiable function $f: \mathbb{R}^{n} \rightarrow \mathbb{R}^{n}$, we consider the diffe- 
rential equation

$$
\dot{x}=f(x) .
$$

Given a regular $k$-dimensional submanifold $M \subset \mathbb{R}^{n}$, let $x^{*} \in M$ be an equilibrium of system (1): $f\left(x^{*}\right)=0$. Then there exists a neighbourhood of $x^{*}$ and $T \in \mathbb{R}^{+}$such that any solution of (1) starting from any point of this neighbourhood is defined at least in $[0, T]$.

For this section we suppose that $M$ is locally positively invariant for system (1) at $x^{*}$, that is, any solution of (1) starting from any point of a neighbourhood of $x^{*}$ in $M$, remains in $M$.

For a given $m \in \mathbb{N}$, let $h: \mathbb{R}^{n} \rightarrow \mathbb{R}^{m}$ be a continuously differentiable function with $h\left(x^{*}\right)=0$. We define an observation system as

$$
\begin{aligned}
& \dot{x}=f(x) \\
& y=h \circ x
\end{aligned}
$$

where $y$ is called the observed function corresponding to solution $x$.

Definition 2.2. We shall say that observation system (1)-(2) is locally observable in $M$ at $x^{*}$, if there exists $\varepsilon \in \mathbb{R}^{+}$with the property that conditions

$$
\begin{aligned}
& \text { for } \underset{\dot{x}^{i}(t)=f\left(\overline{1,2} \underset{\left(x^{i}(t)\right)}{z^{i} \in M,},\left|z^{i}-x^{*}\right|<\varepsilon,[0, T]\right),}{(t \in[0,} \\
& x^{i}(0)=z^{i}, \\
& h\left(x^{1}(t)\right)=h\left(x^{2}(t)\right) \quad(t \in[0, T]),
\end{aligned}
$$

imply

$z^{1}=z^{2}\left(\right.$ and consequently $\left.x^{1}(t)=x^{2}(t) \quad(t \in[0, T])\right)$.

Local observability in $M$ at $x^{*}$ means that, if instead of the solution we can observe a transformation of it, then from this observed function we can recover the solution in a unique way, provided the solution starts at a point of $M$ near the given equilibrium $x^{*}$.

To formulate a sufficient condition which guarantees the local observability of system (1)-(2), we linearize system (1)-(2) at the equilibrium:

$$
L:=f^{\prime}\left(x^{*}\right), \quad C:=h^{\prime}\left(x^{*}\right)
$$

and define

$$
Q:=\left[\begin{array}{llll}
C & C L & \cdots & C L^{n-1}
\end{array}\right]^{T}
$$


Now we recall a theorem proved by Varga (1992), in which in "geometric" terms a sufficient condition is given for local observability.

Theorem 2.3. Suppose that

$$
T_{*} \cap \operatorname{Ker} Q=\{0\}
$$

where $T_{*}$ is the tangent space to $M$ at $x^{*}$. Then system (1)-(2) is locally observable in $M$ at $x^{*}$.

Now we adopt the standard concept of an observer to the case of an observation system with invariant manifold (1)-(2).

Definition 2.4. Given a continuously differentiable function $G: \mathbb{R}^{n} \times \mathbb{R}^{m} \rightarrow$ $\mathbb{R}^{n}$, dynamical system

$$
\dot{z}=G(z, y)
$$

is called a local (exponential) observer for observation system (1)-(2), if the composite system (1)-(2),(4) satisfies the following requirements:

i) If $x(0) \in M$ and $z(0)=x(0)$, then $z(t)=x(t)$ for all $t \in \mathbb{R}^{+}$.

ii) There exists a neighborhood $\mathrm{V}$ of the equilibrium $x^{*}$ such that for all $x(0), z(0) \in V \cap M$, the estimation error $z(t)-x(t)$ tends to 0 (at exponential rate) as $t$ tends to $\infty$.

For the observer design we shall apply the following result (Sundarapandian, 2002):

Theorem 2.5. Suppose that system (1) is Lyapunov stable at equilibrium $x^{*}$, and there exists a matrix $K$ (called gain matrix) such that matrix $L-K C$ is stable. Then system

$$
\dot{z}=f(z)+K[y-h(z)]
$$

is a local exponential observer for observation system (1)-(2).

\section{Evolutionary dynamics for an evolving sexual population}

Following Cressman et al. (1996), we shall consider a large panmictic diploid Mendelian population with alleles $A_{1}, \ldots, A_{n}$, at a single autosomal locus. By assumption, zygotes are produced according to the Hardy-Weinberg proportions. We consider $N$ possible pure phenotypes or behaviour strategies 
identified with the vertices of the standard simplex of the phenotype vectors,

$$
\Delta^{N}=\left\{\left(s_{1}, \ldots, s_{N}\right) \in \mathbb{R}^{N} \mid s_{k} \geq 0 \quad(k \in \overline{1, N}), \sum_{k=1}^{N} s_{k}=1\right\} .
$$

We also suppose that the phenotype of a zygote is uniquely determined by its genotype: for each genotype $A_{i} A_{j}$ let $S_{i j} \in \Delta^{N}$ be the phenotype of an $A_{i} A_{j}$ zygote. (A mixed individual phenotype $s \in \Delta^{N}$ has the usual probabilistic interpretation: $s_{k}$ is the probability for an individual to exhibit the pure phenotype $e_{k}$ ). For each allelic state vector $p \in \Delta^{n}$, the vector

$$
S(p):=\sum_{i, j=1}^{n} p_{i} p_{j} S_{i j}
$$

is the mean phenotype of the population. Given a pay-off matrix, $A \in \mathbb{R}^{N \times N}$, the fitness of an $A_{i} A_{j}$ zygote of a population in allelic state $p \in \Delta^{n}$ is

$$
S_{i j} \cdot A S(p)=\sum_{k, l=1}^{N} S_{i j, k} a_{k l} S_{l}(p)
$$

where $\cdot$ stands for the scalar product of vectors, $S_{i j, k}$ and $S_{l}(p)$ are the $k$-th and the $l$-th components of the vectors $S_{i j}$ and $S(p)$, respectively. Let now $p(t)$ be the allelic frequency vector in the zygote population at time $t$. Then the standard continuous-time evolutionary dynamics for the considered sexual population reads as follows:

$$
\dot{p}_{i}=p_{i}\left(\sum_{j=1}^{n} p_{i} S_{i j}-\sum_{k, l=1}^{n} p_{k} p_{l} S_{k l}\right) \cdot A \sum_{k, l} p_{k} p_{l} S_{k l} \quad(i \in \overline{1, n}) .
$$

For any $p \in \Delta^{n}$ introducing the effective (or marginal) phenotype of allele $A_{i}$,

$$
S^{i}(p):=\sum_{j=1}^{n} p_{j} S_{i j}
$$

for the above dynamics we obtain the following compact form

$$
\dot{p}_{i}=p_{i}\left[S^{i}(p)-S(p)\right] \cdot A S(p) \quad(i \in \overline{1, n}) .
$$

Remark 3.1. It is known that $\Delta^{n}$ and int $\Delta^{n}$ are positively invariant for system (9), furthermore, if for some equilibrium $p^{*} \in \operatorname{int} \Delta^{n}, S\left(p^{*}\right)$ is an evolutionarily stable strategy (ESS), and effective phenotypes are linearly independent 
at $p^{*}$, then $p^{*}$ is asymptotically stable, see Cressman et al. (1996) and also Gámez et al. (2003). For further details on this dynamics we refer to Garay and Varga (1998a), (1998b).

\section{Hopf bifurcation for the evolutionary dynamics}

Now we consider a three-allele system with cyclic dominance $A_{1} \succ A_{2} \succ$ $A_{3} \succ A_{1}$, where the homozygotes exhibit pure phenotypes represented by the canonical basic vectors of $\mathbb{R}^{3}$ :

$$
S_{11}:=e_{1}, \quad S_{22}:=e_{2}, \quad S_{33}:=e_{3}
$$

Then the dominance structure is represented by the hypermatrix

$$
\left[S_{i j}\right]:=\left[\begin{array}{ccc}
e_{1} & e_{1} & e_{3} \\
e_{1} & e_{2} & e_{2} \\
e_{3} & e_{2} & e_{3}
\end{array}\right]
$$

Consider now dynamics (9) with the following payoff matrix: fix an $\varepsilon \in \mathbb{R}$ and define

$$
A(\varepsilon):=\left[\begin{array}{ccc}
-\varepsilon & 1 & -1 \\
-1 & -\varepsilon & 1 \\
1 & -1 & -\varepsilon
\end{array}\right] .
$$

We remind that the payoff matrix $A(\varepsilon)$ defines a generalized "rock-scissorspaper" game in the sense that for $\varepsilon:=0$ it reduces to the payoff matrix of the standard "rock-scissors-paper" game well-known in evolutionary game theory (see e.g. Hofbauer and Sigmund, 1988).

Now the parametrized family of systems corresponding to (9) is

$$
\dot{p}_{i}=p_{i}\left[S^{i}(p)-S(p)\right] \cdot A(\varepsilon) S(p) \quad(i \in \overline{1,3}) .
$$

For a qualitative analysis of this three-dimensional system, by the invariance of the simplex, let us reduce it to a two-dimensional one, substituting $p_{3}=$ $1-p_{1}-p_{2}$ and putting $q:=\left(p_{1}, p_{2}\right)$. The obtained two-dimensional system 
will be written in the compact form

$$
\dot{q}=\varphi(q, \varepsilon) .
$$

An easy calculation shows that for all $\varepsilon \in \mathbb{R}, q^{*}:=\left(\frac{1}{3}, \frac{1}{3}\right)$ is an equilibrium for this system.

Next, we shall prove that in system (13) a Hopf bifurcation occurs at $\varepsilon:=0$. To this end first we recall the following classical theorem (see e.g. Hale and Koçak, 1991).

Theorem 4.1. (Poincaré-Andronov-Hopf) Given an open interval I with $0 \in I$, and an open set $U \subset \mathbb{R}^{2}$ with $0 \in U$, functions $F: I \rightarrow \mathbb{R}^{2 \times 2}$ and $\Phi: U \times I \rightarrow \mathbb{R}^{2}$ such that the right-hand side of the parametrized system

$$
\dot{u}=F(\varepsilon) u+\Phi(u, \varepsilon)
$$

is $C^{3}$. Suppose that

a) for all $\varepsilon \in I$ we have $\Phi(0, \varepsilon)=0$ and $D_{1} \Phi(0, \varepsilon)=0$; and

b) for the eigenvalues $\alpha(\varepsilon) \pm i \beta(\varepsilon)$ of $F(\varepsilon)$ we have $\alpha(0)=0, \beta(0) \neq 0$ and $\alpha^{\prime}(0) \neq 0$.

Then, for any neighbourhood $V$ of 0 in $\mathbb{R}^{2}$, and any $\varepsilon_{0} \in \mathbb{R}^{+}$there exists $\left.\varepsilon_{1} \in\right]-\varepsilon_{0}, \varepsilon_{0}\left[\right.$ such that the differential equation $u=F\left(\varepsilon_{1}\right) u+\Phi\left(\varepsilon_{1}, u\right)$ has a nontrivial periodic orbit in $V$ around the origin.

Applying this theorem to the family of parametrized systems (13), we obtain the following bifurcation result:

Theorem 4.2. For any neighbourhood $W$ of $q^{*}$ in $\mathbb{R}^{2}$, and any $\varepsilon_{0} \in \mathbb{R}^{+}$there exists $\left.\varepsilon_{1} \in\right]-\varepsilon_{0}, \varepsilon_{0}[$ such that the differential equation $\dot{q}=\varphi(q, \varepsilon)$ has a nontrivial periodic orbit in $W$, around equilibrium $q^{*}$.

Proof. For each $\varepsilon \in \mathbb{R}$, let $F(\varepsilon)$ be the Jacobian of $\varphi(\cdot, \varepsilon)$ calculated at the equilibrium, $F(\varepsilon):=D_{1} \varphi\left(q^{*}, \varepsilon\right)$. For any solution $q$ of system (13), define $u:=q-q^{*}$. Then we have

$$
\dot{u}=\varphi(q, \varepsilon)-\varphi\left(q^{*}, \varepsilon\right)=F(\varepsilon) u-F(\varepsilon) u+\varphi(q, \varepsilon) .
$$

Now define

$$
\Phi(u, \varepsilon):=-F(\varepsilon) u+\varphi\left(q^{*}+u, \varepsilon\right),
$$


and take an arbitrary $\varepsilon \in \mathbb{R}$. Since $q^{*}$ is an equilibrium for system (14), we get $\Phi(0, \varepsilon)=0$ and also $D_{1} \Phi(0, \varepsilon)=F(\varepsilon)-F(\varepsilon)=0$. Therefore, conditions a) of Theorem 4.1 are satisfied for the system

$$
\dot{u}=F(\varepsilon) u+\Phi(u, \varepsilon)
$$

obtained from (14). To check condition b) we calculate the Jacobian of (15) to get

$$
F(\varepsilon)=\left[\begin{array}{cc}
\frac{2-2 \varepsilon}{9} & \frac{4}{9} \\
-\frac{4}{9} & -\frac{2+2 \varepsilon}{9}
\end{array}\right] .
$$

For the eigenvalues of $F(\varepsilon)$ we have

$$
\alpha(\varepsilon) \pm i \beta(\varepsilon)=-\frac{2}{9} \varepsilon \pm \frac{3 \sqrt{3}}{9},
$$

implying $\alpha(0)=0, \beta(0) \neq 0$ and $\alpha^{\prime}(0) \neq 0$, so conditions $\left.\mathrm{b}\right)$ of Theorem 10 are also fulfilled. Let $W$ be an arbitrary neighbourhood of $q^{*}$ in $\mathbb{R}^{2}, \varepsilon_{0} \in \mathbb{R}^{+}$ and $\left.\varepsilon_{1} \in\right]-\varepsilon_{0}, \varepsilon_{0}$ [ the parameter value figuring in Theorem 10. Putting $V:=W-q^{*}$, there exists a periodic solution $u$ of system

$$
\dot{u}=F\left(\varepsilon_{1}\right) u+\Phi\left(u, \varepsilon_{1}\right)
$$

in $V$, around the origin, implying $q:=q^{*}+u$ is the required periodic solution of system (13), in $W$ around $q^{*}$.

Example 4.3. From the eigenvalues (16) we see that equilibrium $q^{*}$ is asymptotically stable for $\varepsilon>0$ and unstable for $\varepsilon<0$. Numerical calculations suggest that the above periodic solution is a stable limit cycle, and a supercritical Hopf bifurcation occurs at $\varepsilon:=0$, as shown in Figures 1 and 2. 


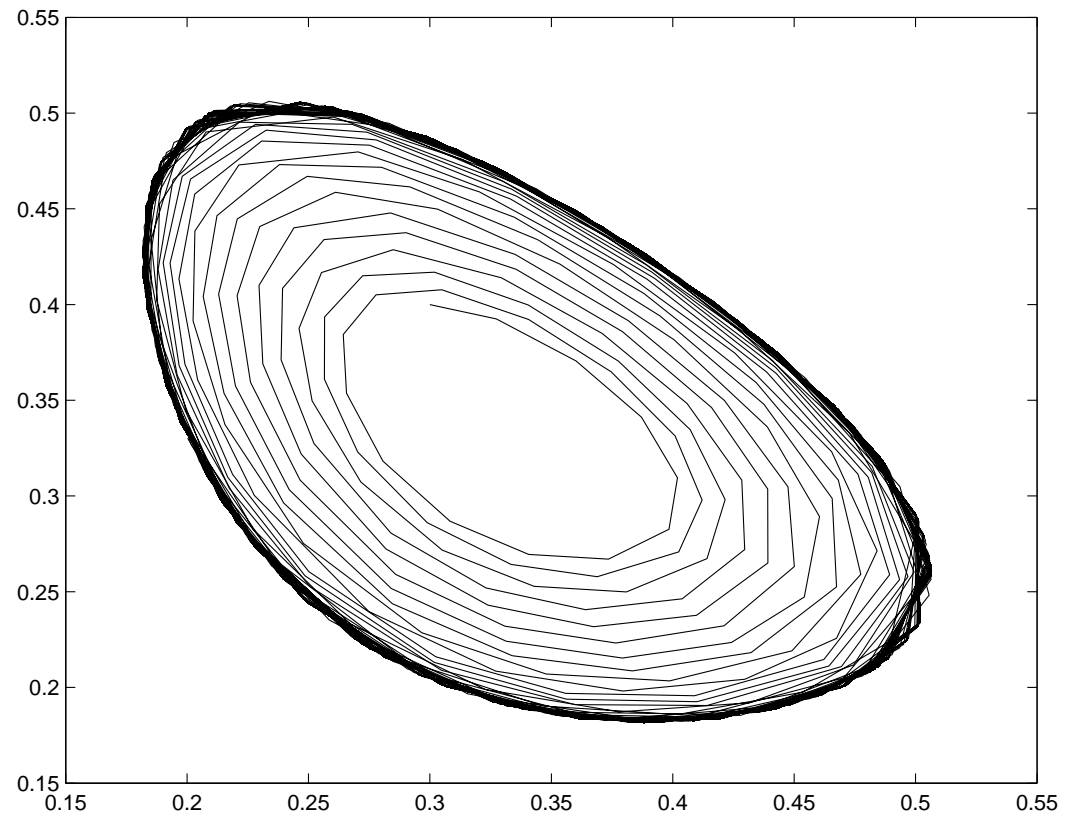

Fig. 1. Periodic orbit for $\varepsilon:=-0.05$, as the $w$-limit set of the solution with initial value $(0.3,0.4)$

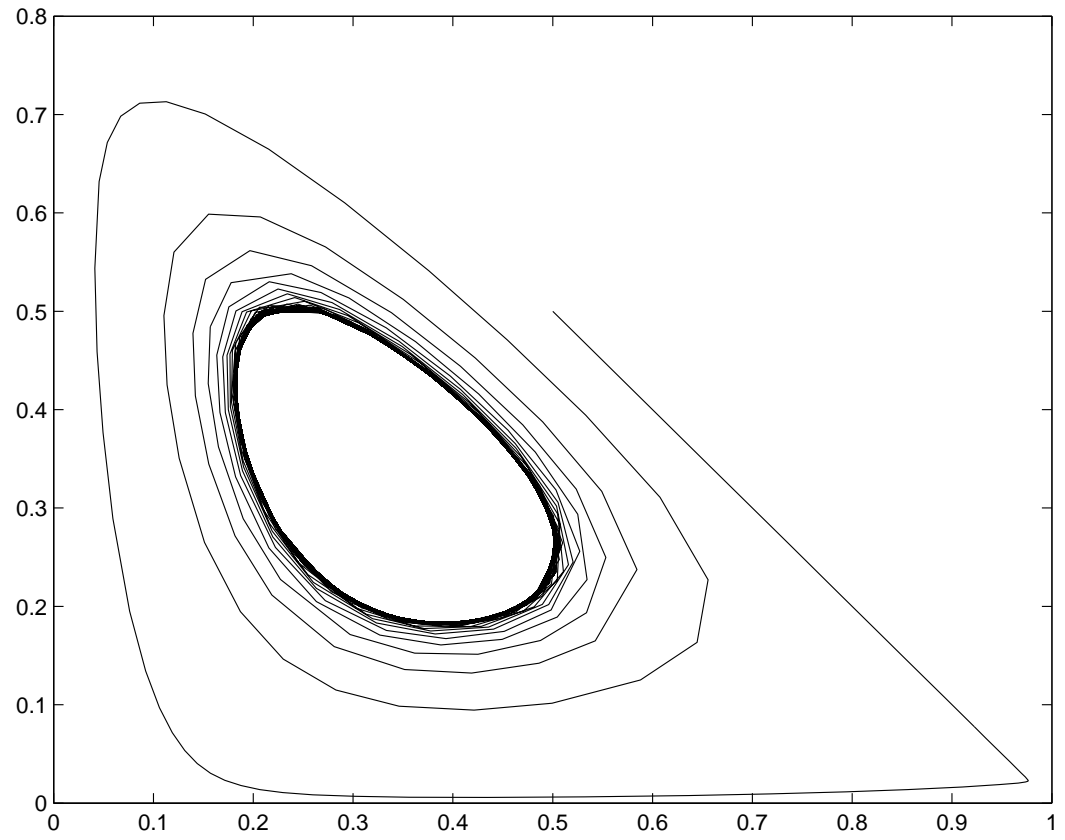

Fig. 2. Periodic orbit for $\varepsilon:=-0.05$, as the $w$-limit set of the solution with initial value $(0.5,0.5)$ 


\section{Phenotypic observation and observer design in an evolving se- xual population}

In this section we will see how to recover the genetic evolution from phenotypic observation, constructing an observer system for two observation situations.

\subsection{Observation of the mean phenotype}

First let us consider the general $n$-dimensional evolutionary dynamics (9)

$$
\dot{p}_{i}=p_{i}\left[S^{i}(p)-S(p)\right] \cdot A S(p) \quad(i \in \overline{1, n}),
$$

and assume we observe the mean phenotype of the population, which means that in terms of the notation of Section 2 we have

$$
h(p):=S(p)-S\left(p^{*}\right) .
$$

(For technical reason the observed quantity is the deviation of the mean phenotype from its equilibrium value). For the application of Theorem 3 we linearize observation system (17)-(18) at equilibrium. Denoting the right-hand side of system (9) by $f$, and using the notation of Section 2, for the linearization we obtain matrices

$$
L:=f^{\prime}\left(p^{*}\right) \quad \text { and } \quad C:=h^{\prime}\left(p^{*}\right) .
$$

The observer we shall construct will not only approximate the solution of the original system, but the solution of the observer is also a substitute of the latter in the sense that the interior of the simplex is long-term invariant for the observer system. We begin with this general statement on the observer constructed for the observation system (17)-(18).

Theorem 5.1. Considering the observation system (17)-(18), suppose that an interior equilibrium $p^{*}$ is asymptotically stable for system (17), for a matrix $K \in \mathbb{R}^{n \times n}$ with some $k_{0} \in \mathbb{R}$ for all $j \in \overline{1, n}, \sum_{i} k_{i j}=k_{0}$ holds, and $L-K C$ is a stable matrix. Then for any solution $p$ of system (17), that is initially close enough to $p^{*}$, int $\Delta^{n}$ is locally long-term invariant for the observer system

$$
\dot{z}=f(z)+K[y-S(z)]
$$


at equilibrium $p^{*}$. (The latter means that for any $z(0)$ from an appropriate neighbourhood of $p^{*}$ in int $\Delta^{n}$, there exists a $t_{0} \in \mathbb{R}^{+}$such that $z(t) \in$ int $\Delta^{n}$ for all $t \in] t_{0}, \infty[$ ).

Proof. By the asymptotic stability of $p^{*}$ we can suppose that $\lim _{\infty} p=p^{*}$. Taking into account that $y$ is the observation corresponding to $p$, let us sum the coordinates of system (19)

$$
\begin{aligned}
\left(\sum_{i} z_{i}\right)^{\prime} & =\sum_{i} f_{i}(z)+\sum_{i} \sum_{j} k_{i j}\left[y_{j}-S_{j}(z)\right]=\sum_{i} f_{i}(z)+\sum_{j}\left[S_{j}(p)-S_{j}(z)\right] \sum_{i} k_{i j} \\
& =\sum_{i} f_{i}(z)+k_{0} \sum_{j}\left[S_{j}(p)-S_{j}(z)\right] .
\end{aligned}
$$

Since $\sum_{i} f_{i}(z)=0$ and $\sum_{j} S_{j}(p)=\sum_{j} S_{j}(z)=1$, we have $\left(\sum_{i} z_{i}\right)^{\prime}=0$, implying $\sum_{i} z_{i}(t)=1$, if $\sum_{i} z_{i}(0)=1$. Moreover, since $\lim _{\infty}(z-p)=0$ and $\lim _{\infty} p=p^{*} \in$ int $\Delta^{n}$, there exists $t_{0} \in \mathbb{R}^{+}$such that $z(t) \in$ int $\Delta^{n}$ for all $t \in] t_{0}, \infty[$.

Example 5.2. Now we illustrate the observer design for the three-allele threephenotype model of Section 4, namely, we consider the dynamic model of the evolution of a sexual population, given by equations (12). Since in this section we shall deal with a fixed value $\varepsilon \in \mathbb{R}^{+}$, in the notation the dependence of the payoff matrix $A$ can be suppressed:

$$
\dot{p}_{i}=p_{i}\left[S^{i}(p)-S(p)\right] \cdot A S(p) \quad(i \in \overline{1,3})
$$

with hypermatrix $\left[S_{i j}\right]$ defined in (10), and matrix $A$ is given in (11). Let us fix $\varepsilon:=0.5$. From the previous section we know that this system has a positive equilibrium $p^{*}:=(1 / 3,1 / 3,1 / 3)$. It is easy to check that for this game, the phenotypic image of equilibrium $p^{*}$, i.e., the mixed phenotype

$$
S^{*}:=S\left(p^{*}\right)=(1 / 3,1 / 3,1 / 3)
$$

is an ESS, see e.g. López, (2003). As an observation the three-dimensional version of (18) is considered:

$$
h(p):=S(p)-S\left(p^{*}\right)
$$


For the matrices of the linearization we obtain

$$
L=\left[\begin{array}{ccc}
-0.0185185 & 0.314815 & -0.12963 \\
-0.12963 & -0.0185185 & 0.314815 \\
0.314815 & -0.12963 & -0.0185185
\end{array}\right]
$$

and

$$
C=2\left[\begin{array}{lll}
S^{* 1} & S^{* 2} & S^{* 3}
\end{array}\right]
$$

where $S^{* 1}, S^{* 2}, S^{* 3}$ are the marginal fenotypes at the equilibrium:

$$
\begin{aligned}
& S^{* 1}=S^{1}\left(p^{*}\right)=(2 / 3,0,1 / 3), \\
& S^{* 2}=S^{2}\left(p^{*}\right)=(1 / 3,2 / 3,0), \\
& S^{* 3}=S^{3}\left(p^{*}\right)=(0,1 / 3,2 / 3) .
\end{aligned}
$$

Hence

$$
C=\left[\begin{array}{ccc}
4 / 3 & 2 / 3 & 0 \\
0 & 4 / 3 & 2 / 3 \\
2 / 3 & 0 & 4 / 3
\end{array}\right]
$$

From the linear independence of vectors (23), by Remark 3.1 we obtain that equilibrium $p^{*}=(1 / 3,1 / 3,1 / 3)$ is asymptotically stable.

Applying Theorem 3, in Gámez et al. (2003) it has been shown that system (20)-(21) is locally observable in int $\Delta^{3}$ at $p^{*}$. In other words, observing the "phenotypic evolution" of the population, in principle we can recover its "genetic evolution", i.e. the state process. For an effective (although approximate) calculation of this state process we apply the observer design method provided by Theorem 2.5.

For

$$
K=\left[\begin{array}{lll}
1 & 0 & 0 \\
0 & 1 & 0 \\
0 & 0 & 1
\end{array}\right]
$$

we obtain that matrix $L-K C$ has eigenvalues with negative real parts. Therefore $L-K C$ is stable and from the linear independence of vectors (23), by 
Remark 3.1 we obtain that equilibrium $p^{*}=(1 / 3,1 / 3,1 / 3)$ is asymptotically stable. Hence by Theorem 2.5 we can construct a local exponential observer for observation system (20)-(21):

$$
\dot{z}_{i}=z_{i}\left(S^{i}(z)-S(z)\right) A S(z)+K[S(p)-S(z)] \quad(i \in \overline{1,3})
$$

By the particular choice of the gain matrix $K$, the conditions of Theorem 5.1 are also satisfied, implying that for any solution $p$ of system (20), that is initially close enough to $p^{*}$, int $\Delta^{n}$ is locally long-term invariant for the observer system (24).

Now we illustrate how the observer approximately provides the required solution from the observed function. Suppose that the initial condition is $p_{0}=$ $(0.3,0.4,0.3)$ for the original system $(20)$ and $z_{0}=(0.35,0.3,0.35)$ for the observer system (24). In the next three figures the coordinates $z_{i}(i \in \overline{1,3})$ of the solution $z$ of the observer system practically end up in the corresponding coordinates $p_{i}(i \in \overline{1,3})$ of the solution $p$ of the original system (20). Since $p^{*}$ is asymptotically stable, if a solution $p$ is initially close enough to $p^{*}$, the "unknown" solution $p$ could also be asymptotically approximated by another solution $w$ of system (20), with the same initial value as $z$. The coordinates $w_{i}(i \in \overline{1,3})$ of this solution are also represented in the figures, showing that the observer performs much better. 


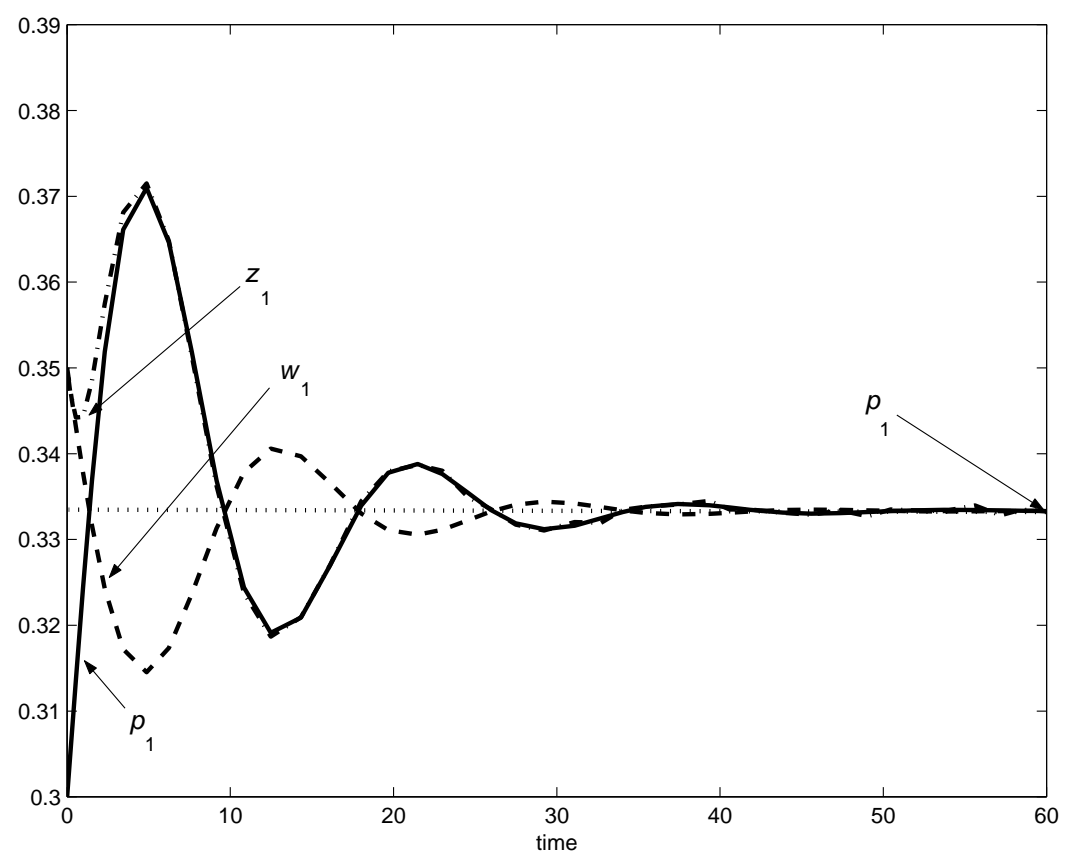

Fig. 3. 1st coordinates of $p, z$ and $w$

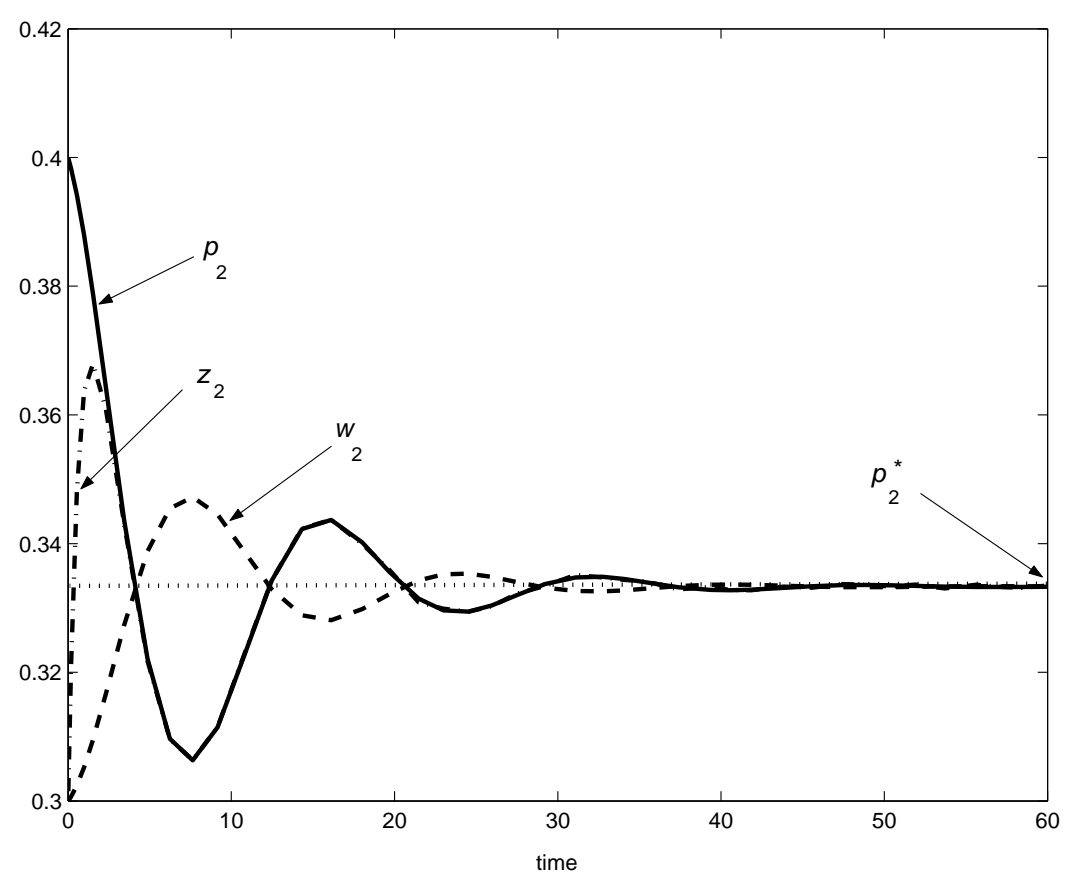

Fig. 4. 2nd coordinates of $p, z$ and $w$ 


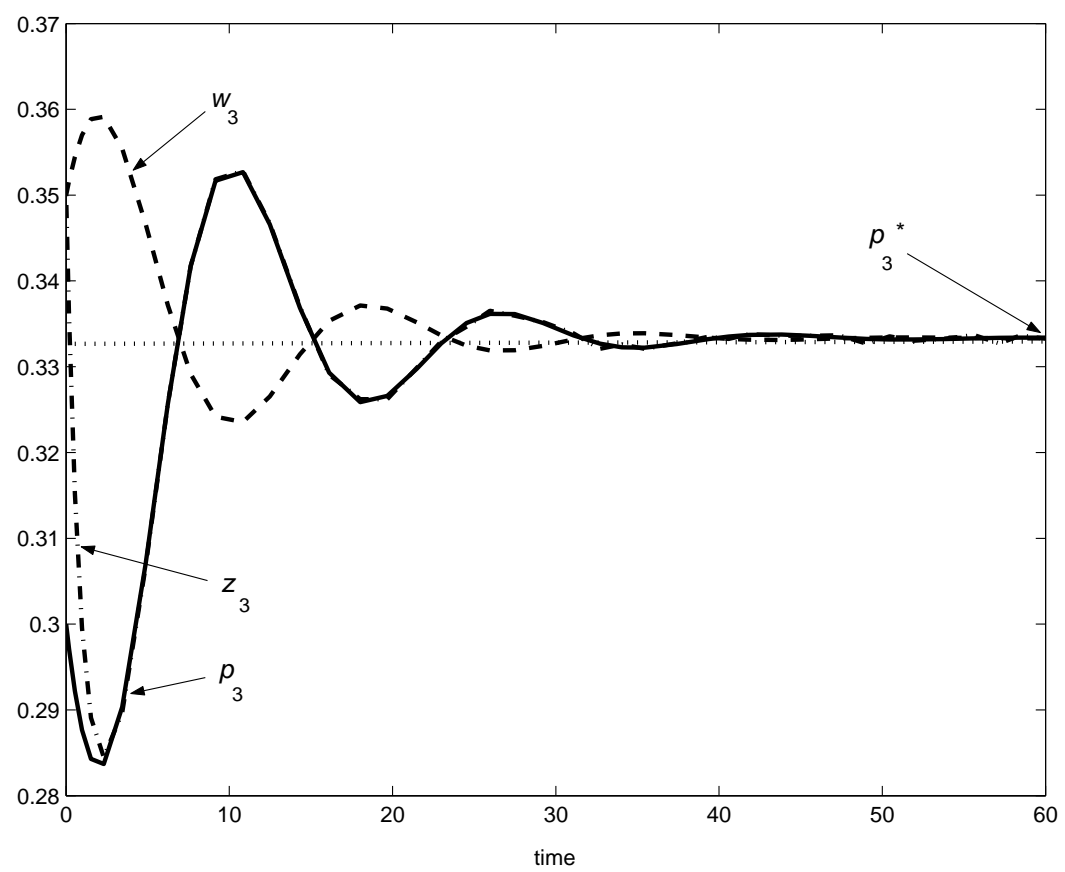

Fig. 5. 3rd coordinates of $p, z$ and $w$

\subsection{Observation of a component of the mean phenotype}

Since in Example 5.2, matrix $C$ is invertible, by the inverse function theorem $h$ is locally invertible at $p^{*}$. This means that near $p^{*}$, solution $p$ can also be calculated from the observed function $y$ by a point-wise inversion. In the following example $h$ is not invertible, so the observer design is the only real option.

Example 5.3. We consider again the evolutionary model (17), fixing again the value of $\varepsilon$ at 0.5 . Let us suppose now that we observe only the first coordinate of the mean phenotype:

$$
h(p):=S_{1}(p)-S_{1}\left(p^{*}\right)
$$

The linearization matrix $L$ is the same as in (22), but now

$$
C:=h^{\prime}(p)=\left[\begin{array}{lll}
4 / 3 & 2 / 3 & 0
\end{array}\right]
$$

Again in Gámez et al. (2003), using transversality condition (3), the local observability of system $(20),(25)$ has been proved. Now we also construct a corresponding observer. Choosing $K:=\left[\begin{array}{lll}0 & 1 & 0\end{array}\right]^{T}$, we obtain that matrix 
$L-K C$ is stable. Applying Theorem 2.5 we obtain the observer system

$$
\dot{z}_{i}=z_{i}\left(S^{i}(z)-S(z)\right) A S(z)+K\left[S_{1}(p)-S_{1}(z)\right] \quad(i \in \overline{1,3}) .
$$

Both for the original system (17) and for the observer (26) we set the same initial conditions as in Example 5.2, $p_{0}=(0.3,0.4,0.3)$, and $z_{0}=(0.35,0.3,0.35)$. The coordinates of the corresponding solutions are presented in Figures 6, 7 and 8 . Figure 9 shows how we can improve the approximation of the third coordinate of $p$, by taking an appropriate gain matrix $K:=\left[\begin{array}{lll}0 & 100 & 0\end{array}\right]^{T}$.

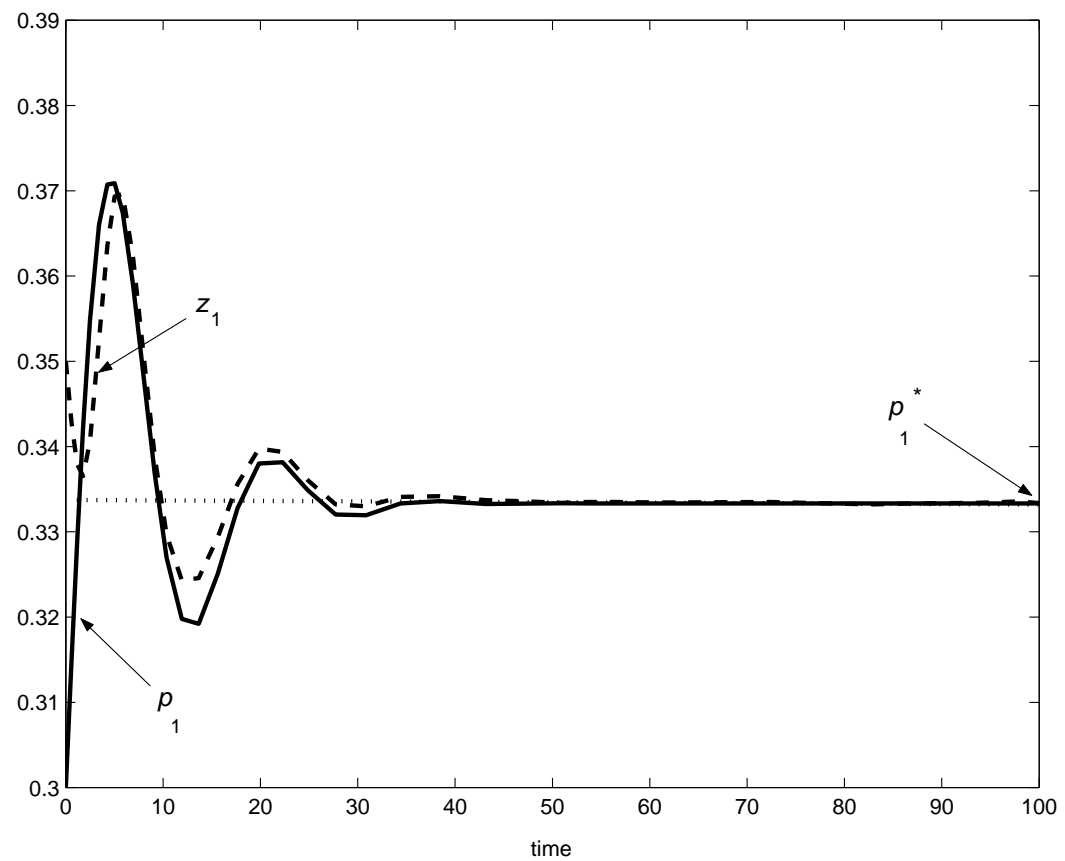

Fig. 6. 1st coordinates of $p$ and $z$, for $K:=\left[\begin{array}{lll}0 & 1 & 0\end{array}\right]$ 


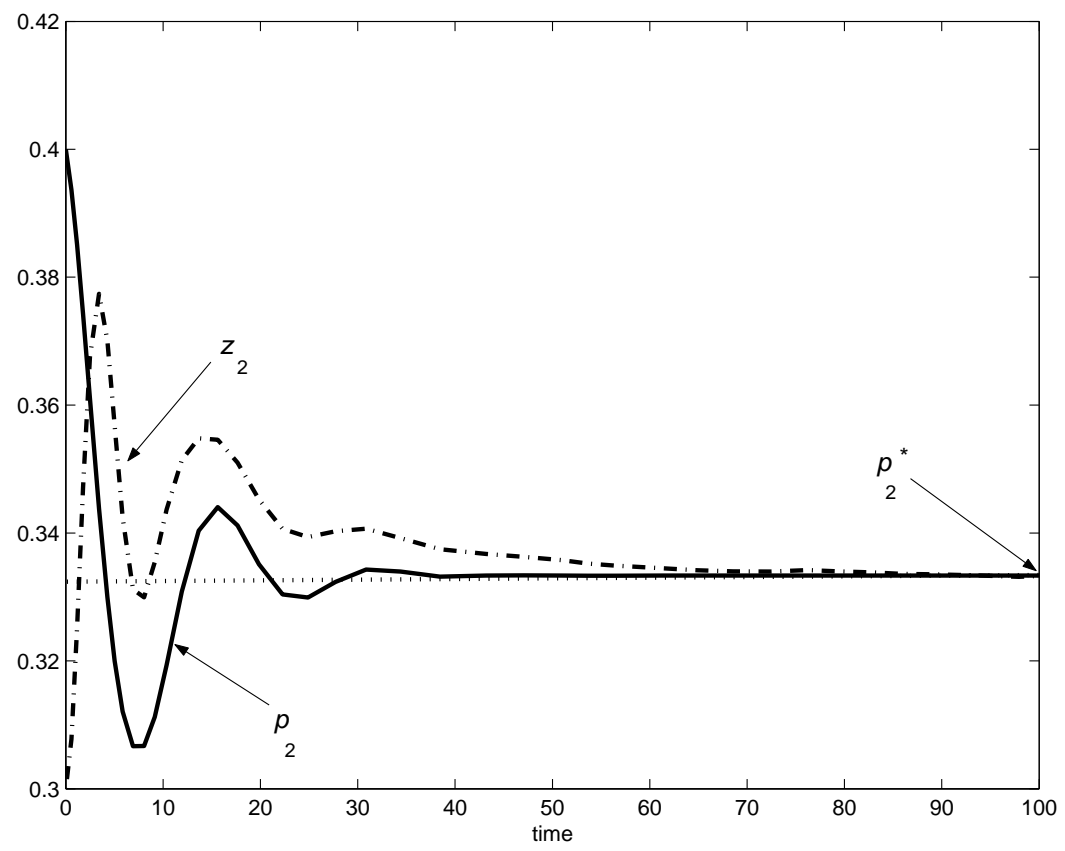

Fig. 7. 2nd coordinates of $p$ and $z$, for $K:=\left[\begin{array}{lll}0 & 1 & 0\end{array}\right]$

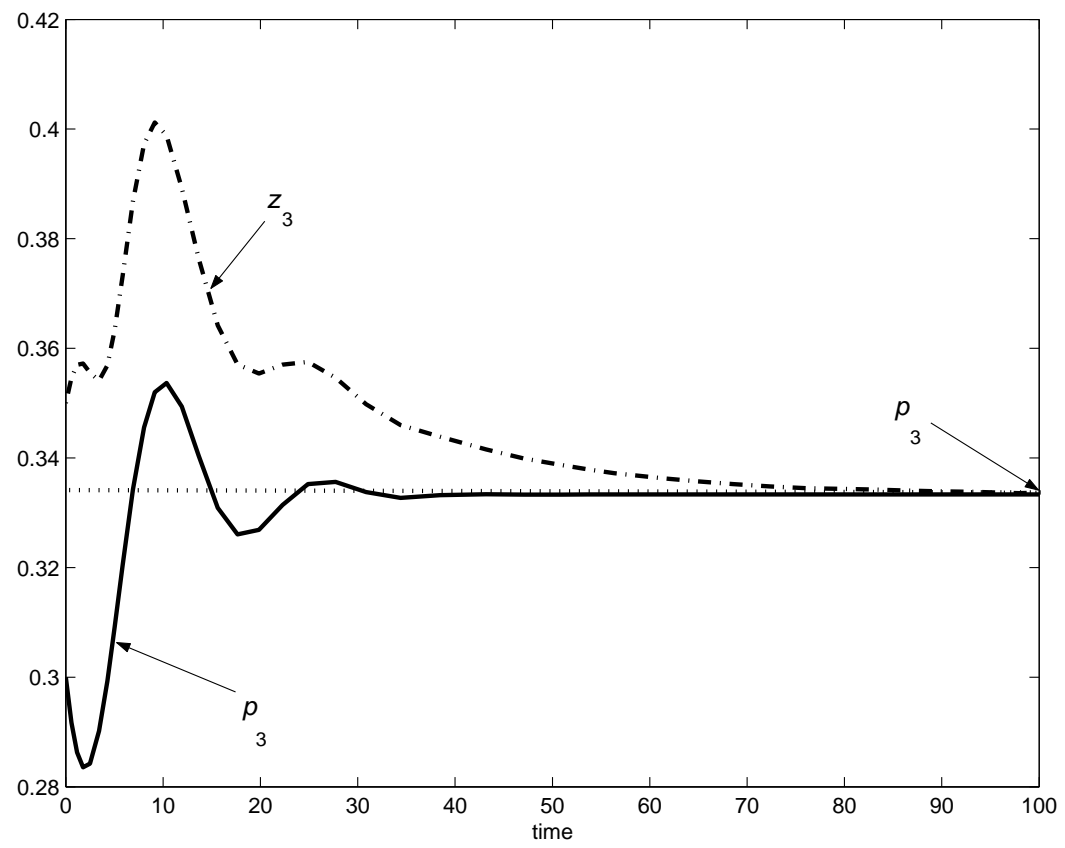

Fig. 8. 3rd coordinates of $p$ and $z$, for $K:=\left[\begin{array}{lll}0 & 1 & 0\end{array}\right]$ 


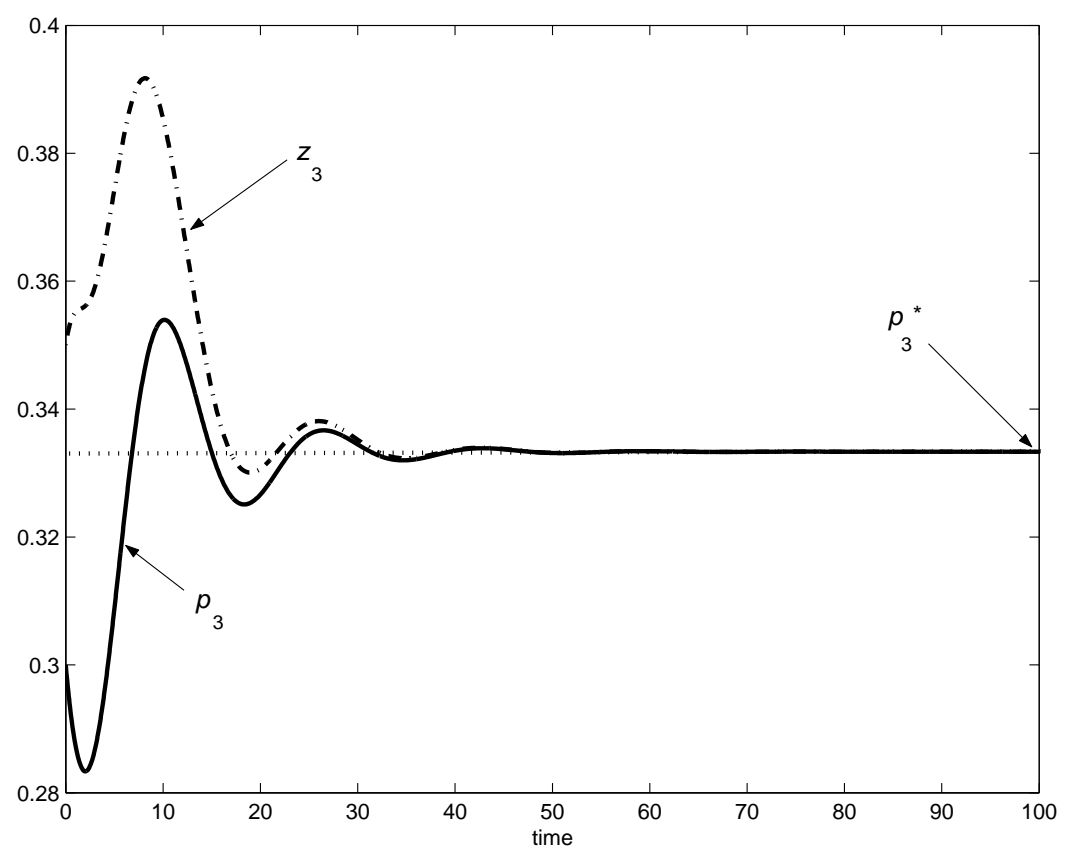

Fig. 9. 3rd coordinates of $p$ and $z$, for $K:=\left[\begin{array}{lll}0 & 100 & 0\end{array}\right]$

\section{Discussion}

The problem of reconstruction of the state process from an observed transform of it, in a natural way arises in the context of the evolution of a sexual population, where certain phenotypic characteristics are observed and the state of the population is described in terms of allele frequencies.

The application of an observer system seems to be an efficient tool for the approximation of an unknown solution, if the corresponding observation is known. In the considered dynamic evolutionary model, in a panmictic Mendelian population a particular type of evolutionary matrix game is played at phenotypic level. In the case of three alleles and three pure phenotypes, reducing the model to a planar vector field, the stability analysis results in a Hopf bifurcation with respect to the parameter of the matrix game. The asymptotic stability obtained for positive parameter values is also important for the observer design.

Numerical results also show how the appropriate choice of the gain matrix the rate of convergence of the asymptotic approximation can be improved. The applied observer design methodology may be also useful for the observation 
analysis and monitoring of different evolutionary and population system models (see e.g. Cressman et al. 2001, Cressman and Garay 2003a, Cressman and Garay 2003b, Garay and Varga 2000, Cressman, et al. 2004, Garay et al. 2003, Cressman and Garay 2006)).

\section{Acknowledgements}

This publication has been realized in the framework of the Hungarian-Spanish intergovernmental scientific and technological collaboration, with the support of the Scientific and Technological Innovation Fund (of Hungary) and the Ministry of Education and Sciences (of Spain), and also supported by the Hungarian National Scientific Research Fund (OTKA T037271).

\section{References}

Cressman, R. and Garay, J., Hofbauer, J. (2001) Evolutionary stability concepts for $N$-species frequency-dependent interactions. J. Theor. Biol. 211, 1-10.

Cressman, R. and Garay, J. (2003a) Evolutionary stability in Lotka-Volterra systems. J. Theor. Biol. 222, 233-245.

Cressman, R. and Garay, J. (2003b) Stability N-species coevolutionary systems. Theor. Pop. Biol. 64, 519-533.

Cressman, R. and Garay, J. (2006) A game-theoretical model for punctuated equilibrium: species invasion and stasis through coevolution. BioSystems $\mathbf{8 4}$, 1-14.

Cressman, R., Hofbauer, J. and Hines, W.G.S. (1996) Evolutionary stability in strategic models of single-locus frequency-dependent viability selection. $J$. Math. Biol., 34, 707-733.

Cressman, R., Krivan, V. and Garay, J. (2004) Ideal Free Distributions, Evo- 
lutionary Games and Population Dynamics in Multiple Species Environments, Am. Nat, 164, 473-489.

Gámez, M., Carreño, R., Kósa, A., Varga, Z. (2003) Observability in strategic models of selection, Biosystems 71, 3, 249-255.

Garay, J., Cressman, R. and Varga, Z., (2003) Optimal behaviour of honey bees based on imitation at fixed densities. Comm. Ecol. 4, 2, 219-224.

Garay, J. and Garay, M., B. (1998) Genetical reachability: When does a sexual population realize all phenotypic states? J. Math. Biol. 37, 146-154.

Garay, J. and Varga, Z. (1998a) Evolutionary stable allele distributions. Journal of Theoretical Biology 191, 163-172.

Garay, J. and Varga, Z. (1998b) When will a sexual population evolve to an ESS? The Proc. Roy. Soc. London Ser. B, 265, 1007-1010.

Garay, J. Varga, Z. (2000) Strict ESS for n-species systems. BioSystems 56: 131-137.

Hale, J. K. and Koçak, H. (1991) Dynamics and Bifurcations. Springer-Verlag.

Hofbauer, J. and K. Sigmund, (1988). The Theory of Evolution and Dynamical Systems. Cambridge University Press, Cambridge.

Lee, E.B. and Markus, L., (1971). Foundations of Optimal Control Theory. Wiley, New York-London-Sydney.

López, I. (2003) Observabilidad y controlabilidad en modelos de evolución. Servicio de Publicaciones de la Universidad de Almería.

López, I., Gámez, M., Garay, J. and Varga, Z. Monitoring in a Lotka-Volterra model, BioSystems (2006a) (in press)

López, I., Gámez, M., Molnar, S. (2006b) Observability and observers in a food web. App. Math. Lett. (submitted) 
Sundarapandian, V. (2002), Local observer design for nonlinear systems, Math. Comp. Mod., 35, 25-36.

Varga, Z., On Observability of Fisher's model of selection, Pure Math. App., Ser. B., 3, 1 (1992), 15-25. 